\title{
Experimental investigation of the travel-time variance of an acoustic wave propagating through the grid-generated turbulence
}

\author{
T. ANDREEVA* and W. DURGIN
}

Mechanical Engineering Department, Worcester Polytechnic Institute, Worcester, Massachusetts, USA

\begin{abstract}
An experimental technique for the investigation of the behaviour of acoustic wave propagation through a turbulent medium is discussed. The present study utilizes the ultrasonic travel-time technique to diagnose a grid-generated turbulence. Travel-time variance is studied versus mean flow velocity, travel distance and outer turbulence scale. The effect of thermal fluctuations, which result in fluctuations of sound speed, is studied using a heated-grid experiment. Experimental data obtained using ultrasonic technique confirm numerical and theoretical predictions of nonlinear increase of the travel-time variance with propagation distance, which could be connected to the occurrence of caustics. The effect of turbulent intensity on the travel-time variance and appearance of caustics is studied. It is demonstrated experimentally that the higher turbulence intensity leads to the shorter distance, at which the first caustic occurs. The probability density for caustics appearance is analysed against the measured wave amplitude fluctuations. The analysis reveals that the region of high-amplitude fluctuations corresponds to the region where the probability of formation of random caustics differs from zero. Experimental results are in very good agreement with theoretical and numerical predictions.
\end{abstract}

\section{Introduction}

The classical theory of acoustic wave propagation through turbulence predicts linear increase of the first-order travel-time variance with the propagation distance [1]. However, recent numerical and theoretical studies exhibit an almost quadratic growth of travel-time variance with travel distance [2-4]. The reason for this behaviour is not entirely understood yet, but it seems to be closely related to the occurrence of caustics [5-8]. If a wave propagates in a random medium, then at some distance $x$ from the source, caustics appear. The information about the distance is important. For example, some tomographic methods used for remote sensing of the atmosphere and the ocean are based on the ray acoustics approach so that knowledge of the distance to the caustics will define the region of applicability of the tomography methods [9-11]. The higher the turbulence intensity, the shorter the distance, at which the first caustic occurs. Codona et al. [5] demonstrated the importance of the occurrence of caustics, when evaluating the average travel time in a random scalar field. The probability of the appearance of the caustics in a random field was explored theoretically $[6,7,12]$ and numerically $[2,3,8,13]$.

The ultrasonic technique for measuring flows offers great potential for turbulent flow diagnostics [14]. The techniques employed have been used in previous work by the current authors [15], wherein a locally isotropic velocity field was considered, which was realized by introducing a grid in a uniform flow [16]. 
In the present study, two features are noteworthy. First, the experimental investigation is performed under well-controlled laboratory conditions. Second, the data acquisition and control systems feature high-speed analogue-digital conversion card that enables excellent resolution of ultrasonic signals. The work aims to investigate the variance of the travel-time fluctuations and probability density function for the appearance of caustics. The measurements cover the regime from weak to strong intensity fluctuations induced by heated and non-heated grids. Comparison with numerical predictions $[2,3]$ as well as theoretical estimations $[1,4]$ supports the study.

\section{Experimental arrangements and results}

In this section, we briefly discuss experimental technique and equipment. A detailed description of the experimental apparatus can be found in $[15,22]$. The experiment falls into two parts. First, for a non-heated grid we perform a study of the travel-time variance as a function of the travel path, turbulent scale and turbulent intensity for long distances. The influence of an integral scale of turbulence is examined by using two grids with different spacing. The turbulent intensity was varied by changing the wind tunnel mean velocity from $0 \mathrm{~m} / \mathrm{s}$ to $20 \mathrm{~m} / \mathrm{s}$. The second set of experiments is conducted for the heated and non-heated grids at constant mean velocity for short travel distances to evaluate the effect of thermal fluctuations on travel-time variances.

\subsection{Non-heated grid experiments}

In the first experimental part of the study, we utilize ultrasonic pulses travelling in straight paths as shown in figure 1 . The sound propagates across a grid-generated turbulence from a transmitter to a receiver separated by a distance $L$ in the direction perpendicular to the mean flow. The travel path $L$ is changed by displacing the transducer-transmitter along a path wave. To ensure the alignment and accurate distance measurement the transmitter receiver is placed in the sliding shaft, as shown in figure 1.

The flowmeter equation can be used to derive an expression for a travel time of a wave travelling from a speaker to a microphone, $t$.

$$
t=\int_{0}^{L} \frac{d y}{c-u} \approx t_{O}+\frac{1}{c^{2}} \int_{0}^{L} u d y, u=U \cos \vartheta+u^{\prime}
$$

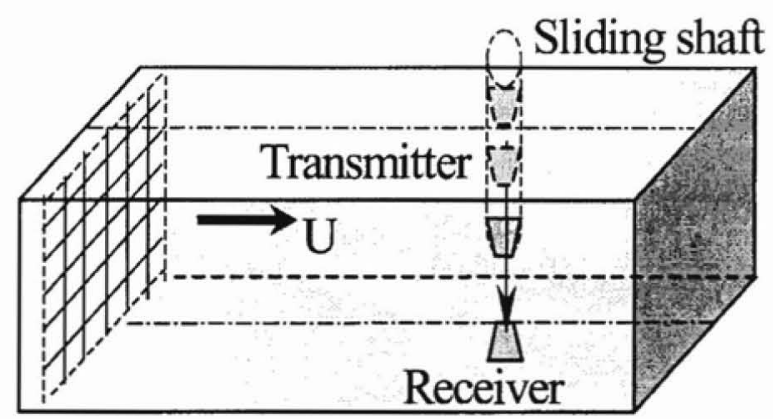

Figure 1. Sketch of the wind-tunnel test section with ultrasonic flowmeter. 
where $t_{0}$ is a travel time in the undisturbed media, $U$ is a mean velocity, $c$ is a sound speed, $u^{\prime}$ indicates fluctuations of the mean flow velocity and $\vartheta$ is an angle between the sound path and a mean velocity vector. In the considered case $\vartheta=90^{\circ}$. In equation (1) we neglected the terms of order $u^{2} / c^{2}$ and higher. In the experiment, the only parameter that is measured is a travel time of ultrasound pulses $t(L)$. The data from experiments performed for a finite number of different lengths $L, L^{\prime}, L^{\prime \prime} \ldots$ are collected. For each of these lengths, a variance of a travel time is calculated as

$$
\sigma_{t}^{2}=\left\langle(t-\langle t\rangle)^{2}\right\rangle
$$

where triangular brackets indicate time averaging.

The experiments were carried out in the $0.3 \mathrm{~m} \times 0.3 \mathrm{~m} \times 1.15 \mathrm{~m}$ test section of a lowturbulence, low-speed, open-circuit-type wind tunnel. Experimental data were obtained for ultrasonic wave propagation downstream of a grid in the wind tunnel. Such grid-generated turbulence is well characterized and features a mean flow with superimposed velocity fluctuations $[16,17]$. The ultrasonic path was perpendicular to the mean flow direction as shown in figure 1. Path length was changed from $0.0508 \mathrm{~m}$ to $0.254 \mathrm{~m}$ and the transducers were of $100 \mathrm{kHz}$ working frequency. The first grid size was $M_{1}=6.35 \times 10^{-3} \mathrm{~m}$. The measurements were collected at $0.63 \mathrm{~m}$ downstream of the grid. The mean flow velocity $U$ was $0 \mathrm{~m} / \mathrm{s}, 10 \mathrm{~m} / \mathrm{s}$, $15 \mathrm{~m} / \mathrm{s}, 18 \mathrm{~m} / \mathrm{s}, 20 \mathrm{~m} / \mathrm{s}$. The corresponding Reynolds numbers $\operatorname{Re}_{M_{1}}$ based on $M_{1}$ were 4200 , $6350,7200,8400$. The same measurements were also made under the same experimental conditions with a different grid that featured mesh size $M_{2}=1.27 \times 10^{-2} \mathrm{~m}$ and the corresponding Reynolds numbers $\operatorname{Re}_{M_{2}}$ based on $M_{2}$ was $8400,12700,14400,16800$. The acoustic wavelength of the transmitted signal, $\lambda_{a} \approx 5.4 \times 10^{-4} \mathrm{~m}$ was selected such that $\lambda_{a}<\lambda_{u^{\prime}}$, where $\lambda_{u^{\prime}} \sim 10^{-2} \mathrm{~m}$ is a wavelength corresponding to the peak of the spectra of longitudinal velocity fluctuations. The same ratio holds for the wavelength corresponding to the maximum of the spectra of temperature fluctuations [22].

Transmitted and received signals in a digital form were transmitted and collected respectively using high-speed data acquisition cards (DAQ). The instant moment of the transmitted wave departure was registered with high precision by National Instrument data DAQ. The received waves were registered/digitized and stored by the CompuScope 82G DAQ with large acquisition memory. Digital representation of the experimental data, provided by the DAQs, allowed determination of the travel time $t$ very precisely. A more detailed description of the experimental particulars may be found in [15]. Figure 2 demonstrates typical data

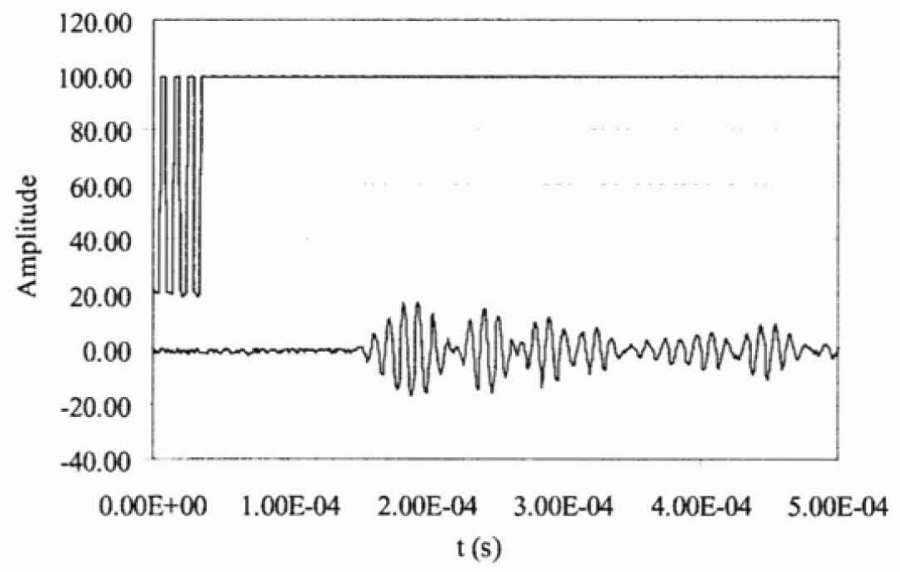

Figure 2. Representation of experimental data obtained from the digital data acquisition system. Square wave corresponds to the transmitted signal and sinusoidal wave represents a received signal. 


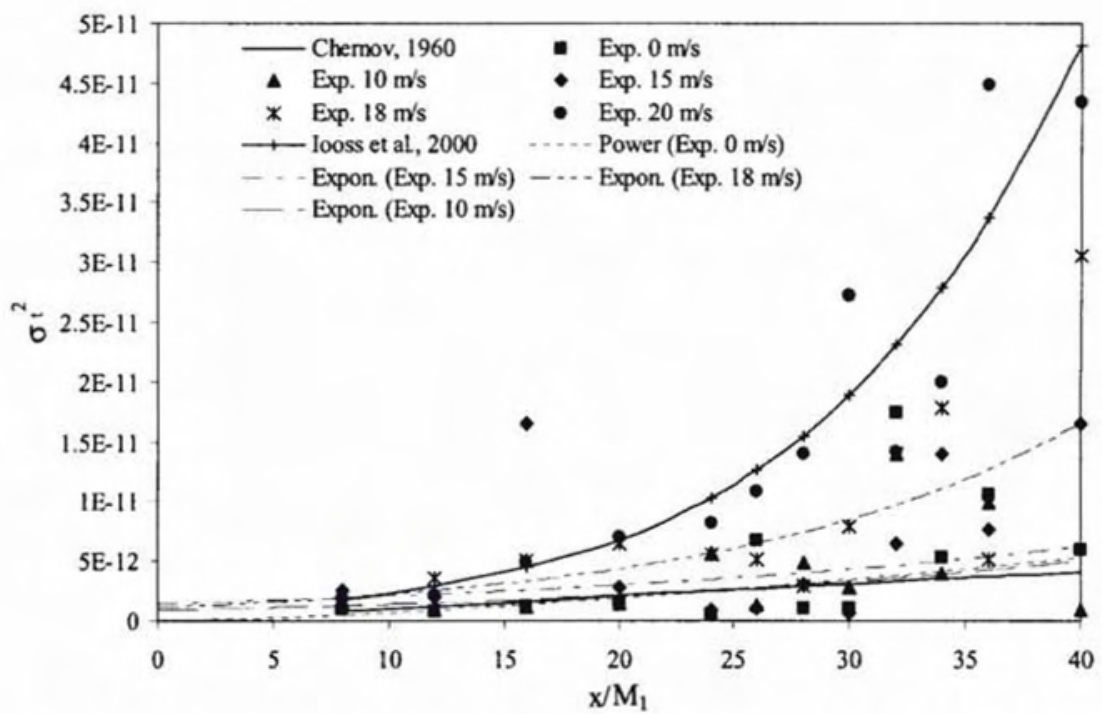

Figure 3. Experimental data for travel-time variance plotted along with theoretical predictions by Chernov [1], and Iooss et al. [4] $M_{1}=6.35 \times 10^{-3} \mathrm{~m}$.

representation obtained from CompuScope $82 \mathrm{G} \mathrm{DAQ}$, transferred to the $\mathrm{PC}$ and processed in Excel. The acquisition rate was $5 \times 10^{7}$ samples/s. Two signals shown in figure 2 are fourpulse burst of square shape waves and a received signal. The transit time for the ultrasound pulse was determined from the correlation function

$$
K_{12}(\tau)=\overline{e_{1}(t+\tau) e_{2}(t)}
$$

where $K_{12}(t)$ is a cross correlation function of $e_{1}$ and $e_{2}$.

In figure 3 the travel-time variances are plotted for the case of $M_{1}=6.35 \times 10^{-3} \mathrm{~m}$ in terms of the normalized distance $x / M_{1}$, where $x$ is a variable travel distance. We compare the travel-time variance with Chernov [1] estimates and with theoretical estimations of secondorder travel-time variance by Iooss et al. [4]. The principle observation is that departures from the Chernov prediction increase with the travel distance for all velocities apart from $0 \mathrm{~m} / \mathrm{s}$ and $10 \mathrm{~m} / \mathrm{s}$. The data for $15 \mathrm{~m} / \mathrm{s}, 18 \mathrm{~m} / \mathrm{s}$ and $20 \mathrm{~m} / \mathrm{s}$ show a trend that appears to be initially linear and finally nonlinear. Travel-time variances corresponding to $0 \mathrm{~m} / \mathrm{s}$ and $10 \mathrm{~m} / \mathrm{s}$ are almost identical with Chernov's prediction, which supports theoretical arguments stating that for phase/travel time the difference ray acoustic approach is accurate enough even beyond the area of the validity of the approach [18]. The travel-time variance corresponding to the $20 \mathrm{~m} / \mathrm{s}$ experiment is in very good correspondence with analytical results predicted by Iooss et al. [4] who developed a theoretical model for the second-order travel-time variance that depicts nonlinear behaviour at large propagation distances. The increase appears linear up to $x / M_{1}=15$ and then travel-time deviations become almost quadratic in growth. Exactly the same dynamics was observed in numerical experiments [4]. Figure 4 provides experimental measurements of the travel-time variances corresponding to the five different velocities plotted along with theoretical predictions for the case of different grid size $M_{2}=1.27 \times 10^{-2} \mathrm{~m}$. In terms of the appearance of nonlinearity, the data in figure 4 exhibit the same tendency as was observed in figure 3 .

Probability densities for the occurrence of caustics were calculated theoretically $[2,6-$ $8,12,19]$. For our experimental data we estimate the probability density of occurrence of caustics using theory developed by Klyatskin [12] and explored by Blanc-Benon et al. [7] and 


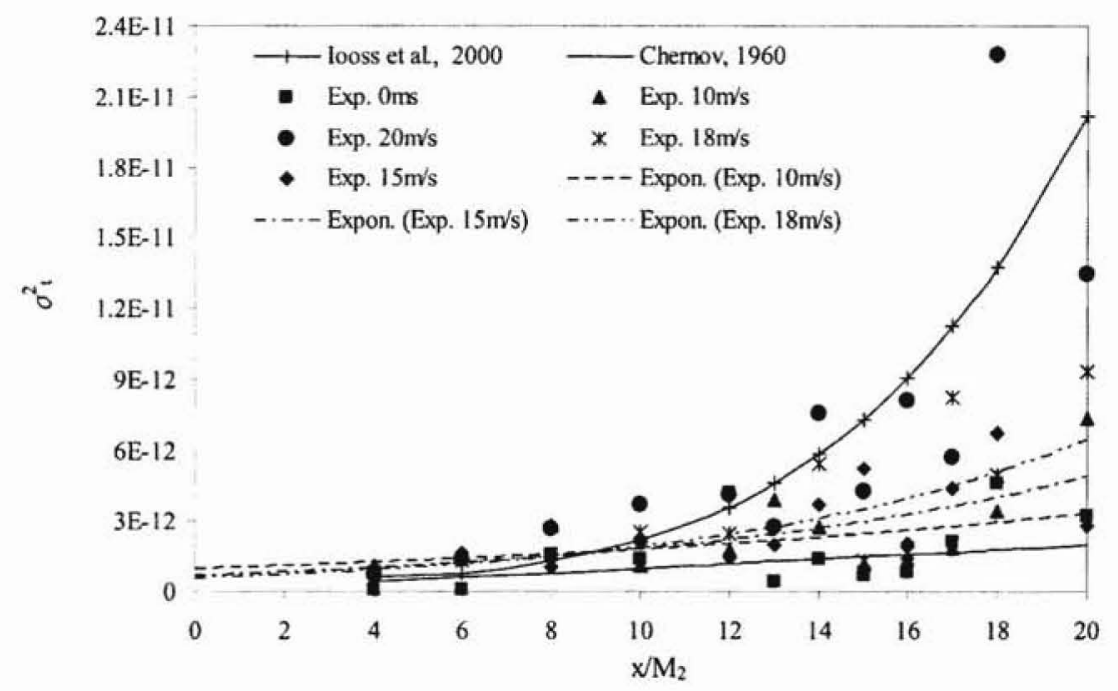

Figure 4. Experimental data for travel-time variance plotted along with theoretical predictions by Chernov [1], and looss et al. [4]. $M_{2}=1.27 \times 10^{-2} \mathrm{~m}$.

Iooss et al. [4]. The probability density function $P(\tau)$ for a plane wave propagating through three-dimensional (3D) isotropic turbulence is defined as $[6,12]$

$$
P(\tau)=\frac{\alpha}{\tau^{4}} \exp \left(-\beta / \tau^{3}\right) ; \alpha=1.74, \beta=0.66
$$

The normalized distance $\tau$ is defined as

$$
\tau=D^{1 / 3} x
$$

where $D$ is the diffusion coefficient introduced by Klyatskin [12] and $x$ is a variable travel distance. For a Gaussian correlation function the diffusion coefficient $D$ in a moving random medium is defined in [7] as

$$
D=\frac{\sqrt{\pi}}{2 L_{0}^{3}} \sigma_{\varepsilon}^{2} ; \sigma_{\varepsilon}=\sqrt{\left\langle\varepsilon^{2}\right\rangle} ; \varepsilon=2 n
$$

where $\sigma_{\varepsilon}$ is a standard deviation of an index of refraction $\varepsilon$. The integral length scale $L_{0}$ is the size of the grid spacing and the downstream development of the integral length scale obeys power law [17]. It is known [20] that fluctuations in the refractive index of a moving medium in the direction of the $x$ axis are given by

$$
n=-\frac{2\left(c^{\prime}+u^{\prime}\right)}{c_{0}}
$$

The ration of turbulent velocity to the mean velocity is $u^{\prime} / U \cdot 100 \% \sim 6 \%$ [22], which is typical for experiments in grid-generated turbulence [16]. In figure 5, the probability density of the occurrence of caustics is plotted along with experimental data for the travel-time variance, Chernov [1] theory for the linear propagation and results of the theoretical model developed by Iooss et al. [4] as functions of normalized distance of propagation, $\tau$ for the grid size $M_{1}=$ $6.35 \times 10^{-3} \mathrm{~m}$. The peak of the probability density function appears at approximately $\tau=$ 0.85 , which corresponds to $x \approx 8.5 \cdot 10^{-2} \mathrm{~m}$, or in non-dimensional units $x / M_{1} \cong 13.2$. The nonlinear behaviour of the travel-time variance appears right after the peak of the probability density function (PDF). In their theoretical modelling, looss et al. [4] detected the maximum 


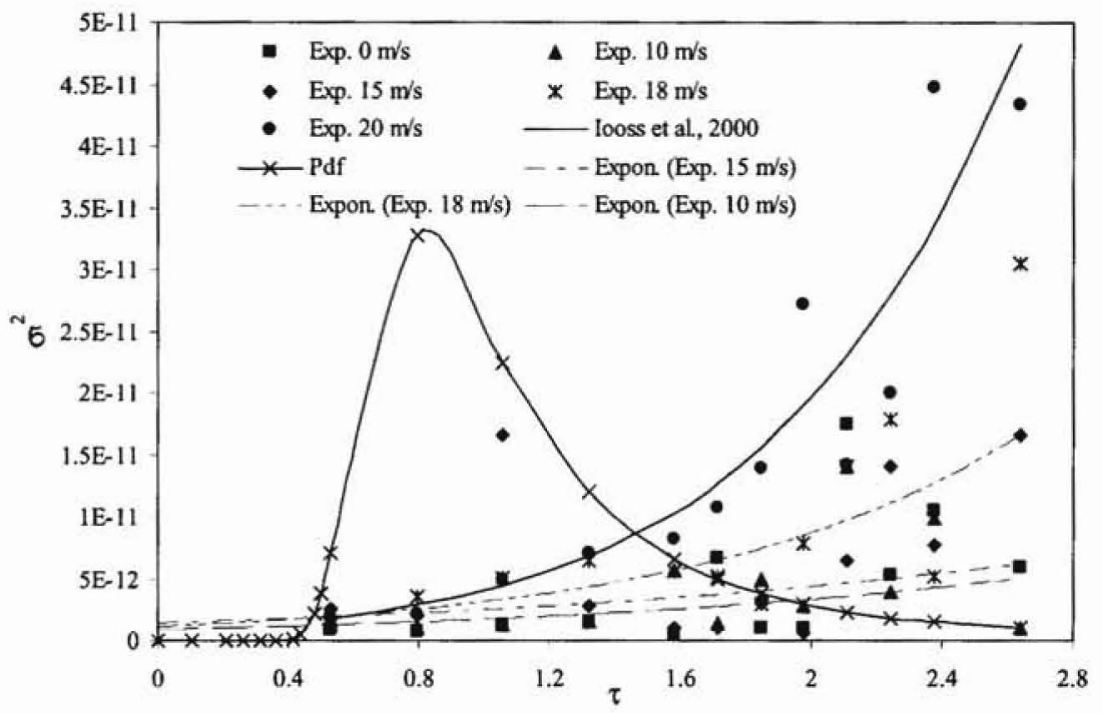

Figure 5. Probability density function of occurrence of caustic along with nonlinear dynamics of the travel-time variance $M_{1}=6.35 \times 10^{-3} \mathrm{~m}$.

value of the probability density with their value $\sigma_{\varepsilon}=0.01$, when $x / M \cong 14.1$. We expected the maximum of the PDF to appear at a shorter distance since standard deviation in the current experiment is greater than one in the theoretical modelling by Iooss et al. [4]. Given the fact that the PDF was recovered based on just 12 discrete points, the prediction is fairly accurate.

Figure 6 provides experimental measurements of the travel-time variances along with the probability density of the occurrence of caustics in the same manner as in figure 4, apart from the grid size $M_{2}=1.27 \times 10^{-2} \mathrm{~m}$. The PDF of the occurrence of caustics obtained for the larger grid exhibits the same tendency as was observed in the first set of experiments for the smaller grid size.

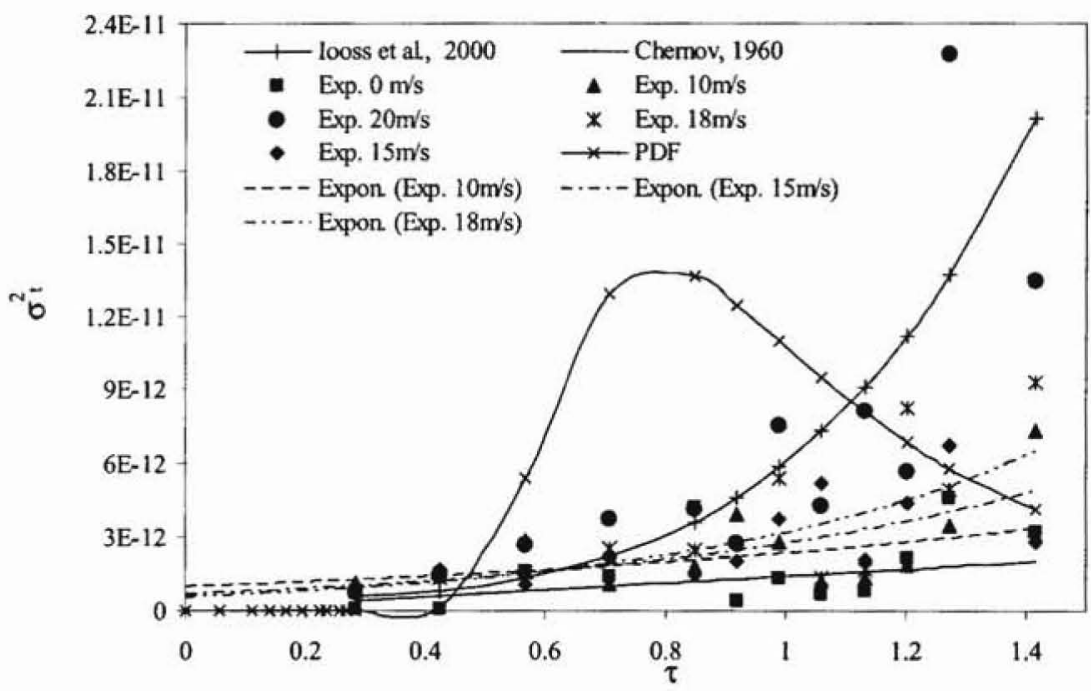

Figure 6. Probability density function of occurrence of caustic along with nonlinear dynamics of the travel-time variance. $M_{2}=1.27 \times 10^{-2} \mathrm{~m}$. 


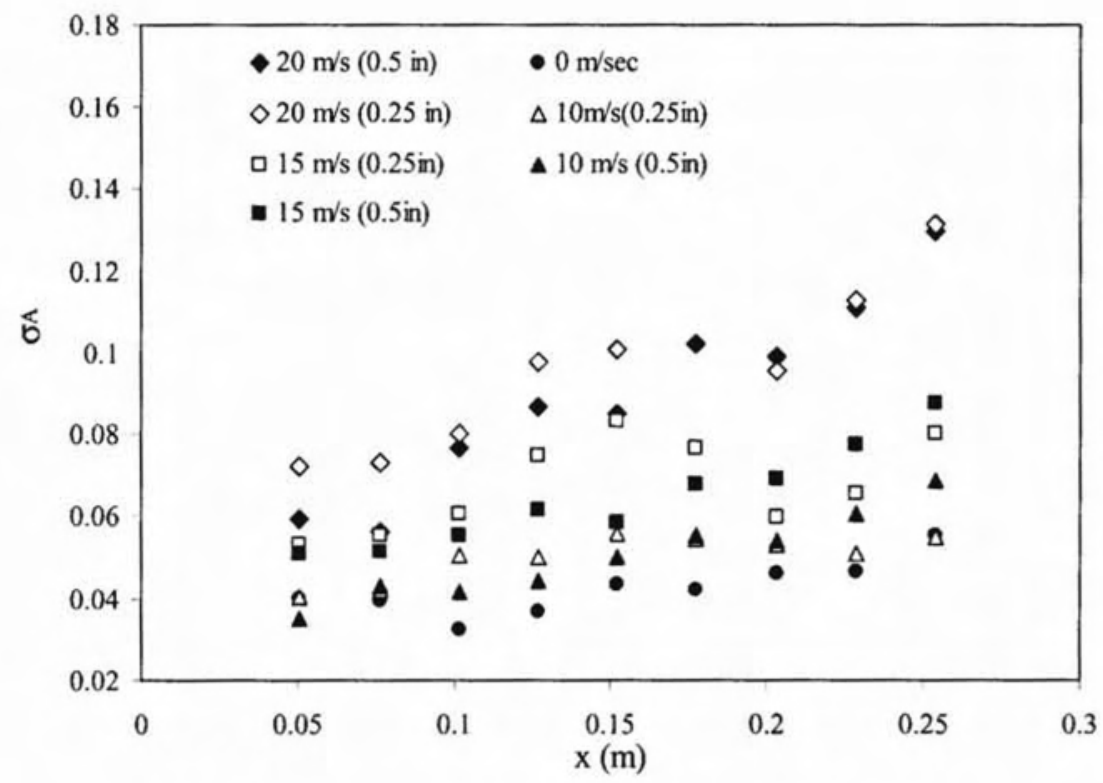

Figure 7. Experimental data for log-amplitude standard deviation.

It is noted in $[19,20]$ that strong fluctuations are connected with random focusing of acoustic waves. We can therefore expect that in a region where $\tau \geq 0.9$ the amplitude fluctuations will be larger than in a region in which travel-time variance is linear. The level of amplitude fluctuations is expressed through the wave amplitude as $\chi=\ln \left(A / A_{0}\right)$ and, consequently, the variance of the log amplitude variations is $\sigma_{A}^{2}=\left\langle(\chi-\langle\chi\rangle)^{2}\right\rangle$. In figure 7 experimental data for the standard deviation of log amplitude variations versus actual travel distance are plotted for undisturbed medium $10 \mathrm{~m} / \mathrm{s}, 15 \mathrm{~m} / \mathrm{s}, 20 \mathrm{~m} / \mathrm{s}$. Experimental data were collected for both, small and large grids to examine the influence of the outer scale. Figure 7 exhibits a substantial increase in standard deviation of log amplitude variations in the region, where probability density is different from zero, which has been predicted theoretically $[19,21]$.

It is difficult to draw a definitive conclusion as for the influence of the outer scale on traveltime variance as well as on the amplitude variations based on this experimental data, since the outer scale is limited to a variation from $6.35 \times 10^{-3} \mathrm{~m}$ to $1.27 \times 10^{-2} \mathrm{~m}$, and because any changes of $L_{0}$ affect other turbulent parameters (turbulent intensity, inner scale).

\subsection{Heated grid experiments}

In this section, experimental data were obtained for ultrasonic wave propagation downstream of a non-heated and heated grid in a wind tunnel for different angular orientations of acoustic waves with respect to the mean flow. The sketch of the experimental set-up is shown in figure 8. Nine cases of different distances $L$ for two different temperatures $T=15^{\circ} \mathrm{C}$ and $T=70^{\circ} \mathrm{C}$, were studied. The grid size was $\mathrm{M}_{3}=2.54 \times 10^{-2} \mathrm{~m}$. To ensure the highquality grid the heating elements were inserted in hollow aluminium rods with diameter $d=6.35 \times 10^{-3} \mathrm{~m}$ defining the solidity of the grid to be 0.36 . The measured temperatures corresponded to the temperature of aluminium rods of the grid. The angle $\beta$ in the figure was changed from 0 to 40 degrees with a 5-degree step. The measurements were collected at $x / M=25^{\prime \prime} / 45^{\prime \prime}$. The mean flow velocity was $U=3.5 \mathrm{~m} / \mathrm{s}$. During the recording of turbulent data the low velocity was chosen in favour of higher velocity in order to maximize the effect of 


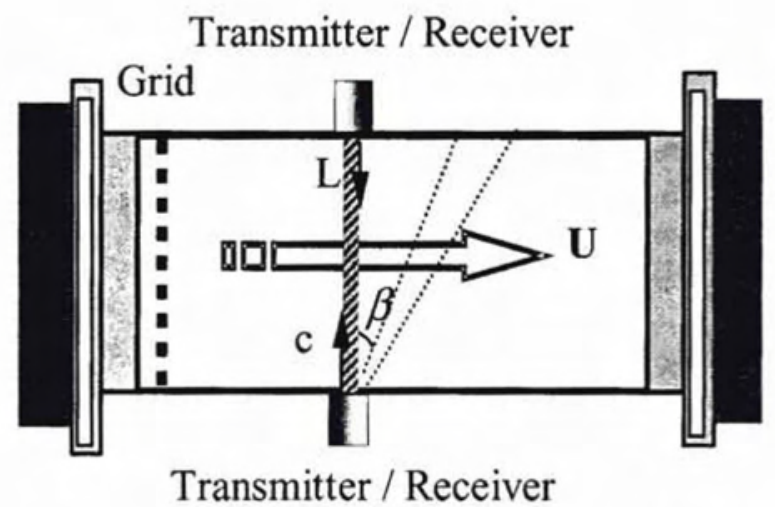

Figure 8. Experimental set-up for the heated grid experiment. $M=2.54 \times 10^{-2} \mathrm{~m}, U=3.5 \mathrm{~m} / \mathrm{s}$.

temperature fluctuations. The Reynolds number $\operatorname{Re}_{M}$ based on $M$ and $U$ was approximately $6 \times 10^{-3}$ and the corresponding Péclet number $P e_{M}=\operatorname{Pr} \operatorname{Re}_{M} \sim 4350 ; \operatorname{Pr}=0.725$ for the working fluid air. For the proposed heated grid experiment, the expected ration of temperature fluctuations to the mean temperature is $\vartheta / T \times 100 \% \sim 1 \% 0$, typical for the particular experimental set-up [16]. The goal is to compare the dynamics of the travel-time variances for heated and non-heated grid experiments with all other experimental conditions being the same.

Figure 9 demonstrates results for the travel-time variance obtained from the experiment conducted at $T=15^{\circ} \mathrm{C}$. The travel distance is merely a half of the distance covered in the first series of the experiments described in section 2.1. The Chernov [1] solution predicts a linear growth of time variance with distance of propagation. Clearly, for the small distances the presented experimental data confirm that result $[22,23]$.

With the same field characteristics as above but the temperature of the grid, which was set at $T=70^{\circ} \mathrm{C}$ for this experiment, we carried out a second set of experiments to demonstrate the

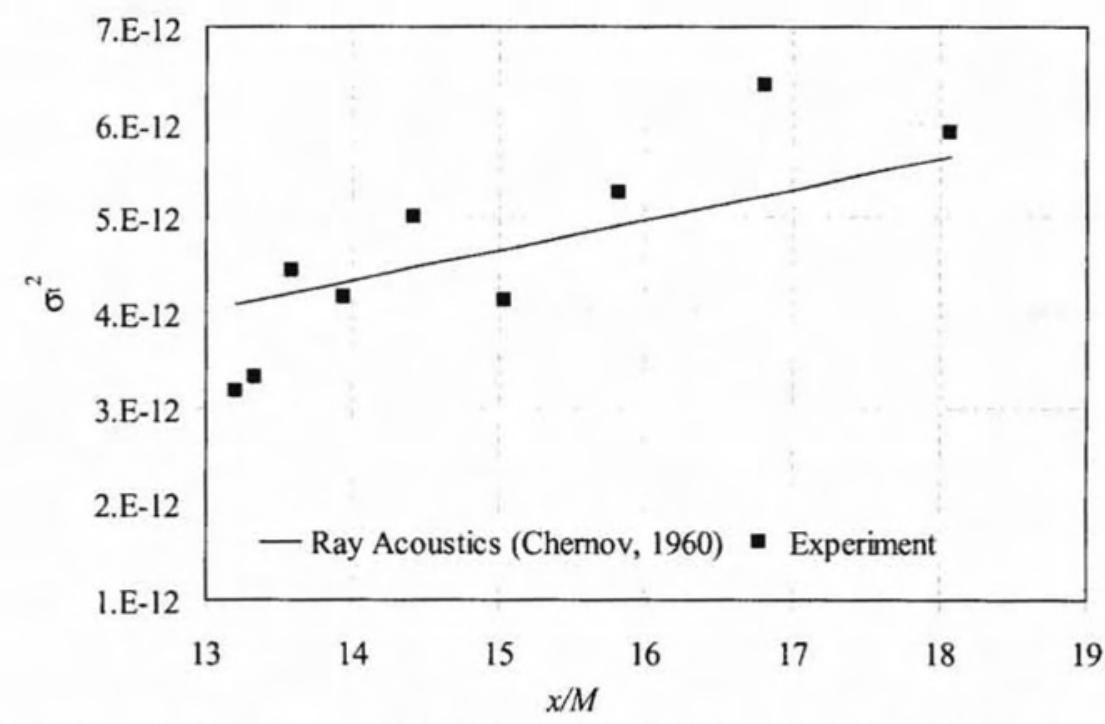

Figure 9. Experimental data for the travel-time variance versus normalized travel distance. Ray acoustics solution is plotted for a comparison. $T=15^{\circ} \mathrm{C}, M=2.54 \times 10^{-2} \mathrm{~m}, U=3.5 \mathrm{~m} / \mathrm{s}$. 


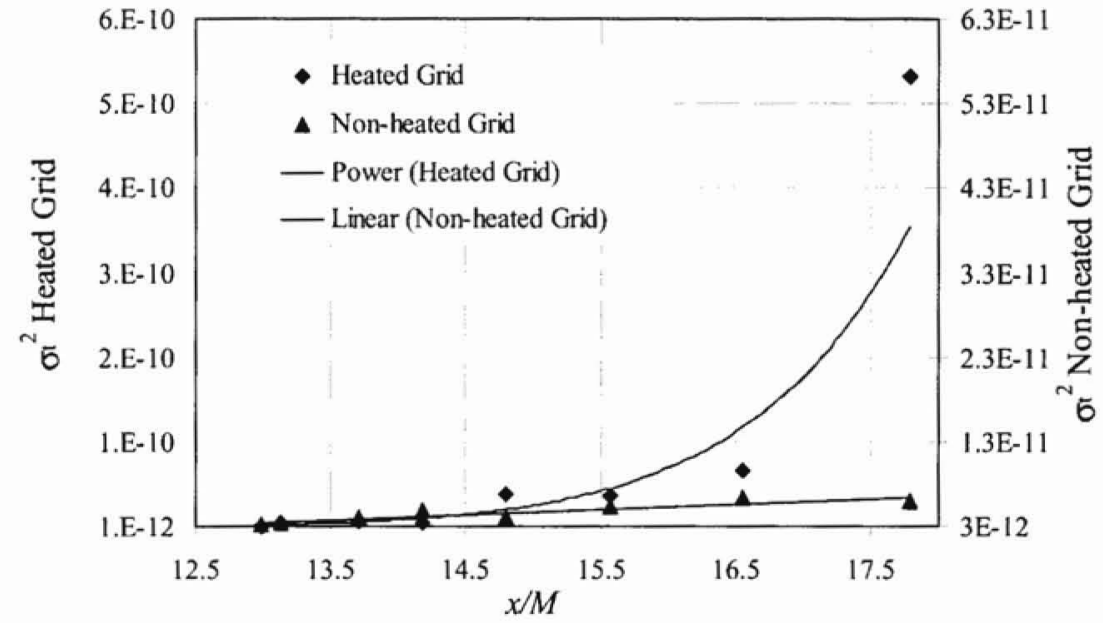

Figure 10. Experimental data for the travel-time variance versus normalized travel distance. $T=15^{\circ} \mathrm{C}, T=70^{\circ} \mathrm{C}$, $M=2.54 \times 10^{-2} \mathrm{~m}, U=3.5 \mathrm{~m} / \mathrm{s}$.

effect of temperature fluctuations. Figure 10 shows the results, again the travel-time variation as a function of scaled distance of propagation. The variance obtained for two values of the turbulent field standard deviation is $\sigma \approx 3 \times 10^{-3}$ for the non-heated grid experiment and $\sigma \approx 1$ for the heated grid experiment. It is obvious that temperature fluctuations lead to the appearance of nonlinearity in the dynamics of the travel-time variance. The travel-time variance at $T=15^{\circ} \mathrm{C}$ is plotted for a comparison. The experimental outcome presented in figure 10 is well expected in accordance with theoretical and numerical predictions [2, 3, 6, $8,12,24]$. The experimental data confirm theoretically and numerically established theory stating that the higher the turbulent intensity, the shorter the distance at which the first caustics occur [2]. The curve for the case of heated grid travel-time variance presents the shapes predicted theoretically and numerically. It therefore follows that when $\sigma \approx 1$ the probability of appearance of the caustics becomes comparable with unity.

\section{Conclusions}

Experimental data for travel time and wave amplitude have been collected using the ultrasonic technique in an inhomogeneous moving medium produced by heated and non-heated grids placed at the beginning of a wind tunnel test section. We have presented results for the travel time variance and the standard deviation of log-amplitude variations.

Experimental data confirm numerical and theoretical predictions of a nonlinear increase of the travel-time variance with propagation distance. To reinforce this point of view the probability density function associated with the occurrence of the first caustic specific for our experimental conditions has been plotted against the travel-time variation versus nondimensional distance. The measurements of the wave amplitude confirm theoretical/numerical predictions that the region of strong amplitude fluctuations coincides with the region where the probability of formation of random caustics is different from zero [19].

The effect of thermal fluctuations superimposed on velocity fluctuations has been studied in the heated-grid experiment. For heated and non-heated grid experiments it has been demonstrated that, after a linear increase (Chernov approximation), the travel-time variance departs from linear behaviour at certain propagation distance, thought to be caused by the 
occurrence of caustics. Experimental data from the heated grid experiments have confirmed theoretically/numerically based theory stating that growth of turbulent field intensity leads to the development of nonlinearity and caustics even at short distances. It has been shown that nonlinearity or caustics unavoidably occur at a large distance in the case of a weak turbulent field (standard deviation, $\sigma \ll 1$ ) or at shorter distances at the presence of strong fluctuations $(\sigma \sim 1)$.

\section{References}

[1] Chernov, L., 1961, Wave Propagation in a Random Medium (New York: McGraw-Hill).

[2] Juvé, D., Blanc-Benon, Ph. and Comte-Bellot, G., 1991, Transmission of acoustic waves through mixing layers and 2D isotropic turbulence. Turbulence and Coherent Structures (Métais and Lesieur (eds)). Selected papers from Turbulence 89: Organized Structures and Turbulence in Fluid Mechanics, Grenoble, 18-21 September.

[3] Karweit, M., Blanc-Benon, Ph., Juvé, D. and Comte-Bellot, G., 1991, Simulation of the propagation of an acoustic wave through a turbulent velocity field: a study of phase variance. Journal of Acoustical Society of America, 89, 52-62.

[4] Iooss, B., Blanc-Benon, $\mathrm{Ph}$. and Lhuillier, C., 2000, Statistical moments of travel times at second order in isotropic and anisotropic random media. Waves in Random and Complex Media, 10, 381-394.

[5] Codona, J. L., Creamer, D. B., Flatte, S. M., Frelich, R. G. and Henyey, F. S., 1985, Average arrival time of wave pulses through continuous random media. Physics Review Letters, 55, 9-12.

[6] Kulkarny, V. A. and White, B. S., 1982, Focusing of waves in turbulent inhomogeneous media. Journal of Physics of Fluids, 25, 1779-1784.

[7] Blanc-Benon, Ph., Juvé, D., Ostashev, V. E. and Wandelt, R., 1995, On the appearance of caustics for plane sound-wave propagation in moving random media. Waves in Random and Complex Media, 5, 183-199.

[8] Blanc-Benon, Ph., Juvé, D. and Comte-Bellot, G., 1991, Occurrence of caustics for high-frequency caustic waves propagating through turbulent field. Theoretical and Computational Fluid Dynamics, 2, 271-278.

[9] Iooss, B. and Galli, A., 2000, Statistical tomography for seismic reflection data. Proceedings of the 6th International Geostatistics Congress, Cape Town, South Africa, April.

[10] Spiesberger, J. L., 1985, Ocean acoustic tomography: travel time biases. Journal of Acoustical Society of America, 77, 83-100.

[11] Samueldis, Y. and Mukerji, T., 1998, Velocity shift in heterogeneous media with anisotropic spatial correlation. International Geophysical Journal, 134, 778-786.

[12] Klyatskin, V. I., 1993, Caustics in random media. Waves in Random and Complex Media, 3, 93-100.

[13] Blanc-Benon, Ph., Juvé, D. and Chevret, P., 1995, On the influence of the turbulence modeling for atmospheric sound propagation. Journal of Acoustical Society of America, 98, 2924.

[14] Lynnworth, L. C., 1989, Ultrasonic Measurements for Process Control (San Diego: Academic Press).

[15] Andreeva T. A. and Durgin, W. W., 2003, Ultrasound technique for prediction of statistical characteristics of grid-generated turbulence. AIAA Journal, 41, 1438-1443.

[16] Yeh, T. T. and van Atta, C. W., 1973, Spectral transfer of scalar and velocity fields in heated-grid turbulence. Journal of Fluid Mechanics, 58, 233-261.

[17] Sreenivasan, K. R., Tavoularis, S., Henry, R. and Corrsin, S., 1980, Temperature fluctuations and scales in grid-generated turbulence. Journal of Fluid Mechanics, 100, 597-621.

[18] Rytov, S. M., Kravtsov, Yu. A. and Tatarskii, V. I., 1987, Elements of Random Process Theory: Principles of Statistical Radiophysics, Vol. 4 (Berlin: Springer-Verlag).

[19] Kravtsov, Yu. A., 1969, Strong fluctuations of the amplitude of a light wave and probability of formation of random caustics. Soviet Physics JETP, 28, 413-414.

[20] Tatarski, V. I., 1971, The Effect of the Turbulent Atmosphere on Waves Propagation (Jerusalem: Israel Program for Scientific Translation).

[21] Blanc-Benon, Ph. and Juvé, D., 1993, Intensity fluctuations of spherical acoustic waves propagating through thermal turbulence. Waves in Random and Complex Media, 3, 71-83.

[22] Andreeva T. A., 2003, Ultrasonic technique in determination of grid-generated turbulent flow characteristics. $\mathrm{PhD}$ Dissertation, Worcester Polytechnic Institute.

[23] Andreeva, T. A. and Durgin, W. W, 2004, Experimental investigation of ultrasound propagation in turbulent, diffractive media. The Journal of the Acoustical Society of America, 115, 1532-1536.

[24] Snieder, R. and Sambridge, M., 1993, The ambiguity in ray perturbation theory. Journal of Geophysical Research, 98, 22 021-22034. 